\title{
EQUIVALENCE OF HAAR BASES ASSOCIATED TO DIFFERENT DYADIC SYSTEMS
}

\author{
HUGO AIMAR, ANA BERNARDIS, AND LUIS NOWAK
}

\begin{abstract}
In this note we give sufficient conditions on two dyadic systems on a space of homogeneous type in order to obtain the equivalence of corresponding Haar systems on Lebesgue spaces. The main tool is the vector valued Fefferman-Stein inequality for the Hardy-Littlewood maximal operator.
\end{abstract}

\section{INTRODUCTION}

The usual orthonormal Haar system $\mathcal{H}$ in $L^{2}(\mathbb{R})$ given by $h_{k}^{j}(x)=2^{j / 2} h\left(2^{j}-k\right)$, $j, k \in \mathbb{Z}$, where $h(x)=\chi_{[0,1 / 2)}(x)-\chi_{[1 / 2,1)}(x)$, is only the best known of a large family of Haar type bases in one dimension. Of course the family of dyadic intervals $I_{k}^{j}=\left[k 2^{-j},(k+1) 2^{-j}\right), k \in \mathbb{Z}, j \in \mathbb{Z}$ is the natural dyadic family associated to $\mathcal{H}$ in the sense that $I_{k}^{j}$ is the set where $h_{k}^{j}$ does not vanish. Moreover each $h_{k}^{j}$ is constant on the two subintervals $I_{2 k}^{j+1}$ and $I_{2 k+1}^{j+1}$ of $I_{k}^{j}$. Notice that $\mathcal{H}$ is not the only system satisfying all the above properties. Moreover, any system of the form $\tilde{\mathcal{H}}=\left\{\psi_{k}^{j}=w_{k}^{j} h_{k}^{j}: k \in \mathbb{Z}, j \in \mathbb{Z}\right\}$ with $w_{k}^{j}= \pm 1$ is an orthonormal basis in $L^{2}(\mathbb{R})$, each function $\psi_{k}^{j}$ has $I_{k}^{j}$ as its essential support and each $\psi_{k}^{j}$ is constant on each member of the offspring of $I_{k}^{j}$.

We are interested in the equivalence of different Haar bases built on different dyadic systems on spaces of homogeneous type. It is easy to construct simple perturbations of the dyadic intervals in $\mathbb{R}$, or of the dyadic cubes in $\mathbb{R}^{n}$, as images through bi-Lipschitz functions of the standard dyadic intervals. Precisely, is $F$ is an one to one mapping from $\mathbb{R}^{n}$ onto $\mathbb{R}^{n}$, and $Q_{\vec{k}}^{j}=\prod_{i=1}^{n} I_{k_{i}}^{j}$, with $\vec{k}=\left(k_{1}, \ldots, k_{n}\right) \in \mathbb{Z}^{n}$ and $j \in \mathbb{Z}$, the family $\left\{F\left(Q_{\vec{k}}^{j}\right): j \in \mathbb{Z}, \vec{k} \in \mathbb{Z}^{n}\right\}$ satisfies the basic properties of the usual dyadic cubes. Moreover, if $F$ is bi-Lipschitz; that is, $c_{1}|x-y| \leq$ $|F(x)-F(y)| \leq c_{2}|x-y|$ for two positive constant $c_{1}$ and $c_{2}$ and every $x, y$ in $\mathbb{R}^{n}$, then the metric control for the new "cubes" $F\left(Q_{\vec{k}}^{j}\right)$ is preserved. In fact, for some fixed constants $\alpha_{1}$ and $\alpha_{2}$, that depend only on $n$ and $F$, we have that $B\left(F(y), \alpha_{1} 2^{-j}\right) \subseteq F\left(Q_{\vec{k}}^{j}\right) \subseteq B\left(F(y), \alpha_{2} 2^{-j}\right)$ for some $y \in Q_{\vec{k}}^{j}$, every $j \in \mathbb{Z}$ and every $\vec{k} \in \mathbb{Z}^{n}$. It seems somehow natural to expect that in some sense a Haar system in $L^{2}\left(\mathbb{R}^{n}\right)$ supported by the family $\left\{F\left(Q_{\vec{k}}^{j}\right): j \in \mathbb{Z}, \vec{k} \in \mathbb{Z}^{n}\right\}$ should be equivalent to a Haar system supported by the family $\left\{Q_{\vec{k}}^{j}: j \in \mathbb{Z}, \vec{k} \in \mathbb{Z}^{n}\right\}$. A much more general setting is provided by the remarkable construction given by M. Christ in [3], which we shall describe with some detail in Section 5 .

2000 Mathematics Subject Classification. 42C15, 42B20, 28C15 .

Key words and phrases. Haar basis, equivalence of bases, spaces of homogeneous type. 
Dyadic type families of subsets in metric or quasi-metric measure spaces can be constructed by elementary disjunction of balls. When only the covering and nesting properties of dyadic sets are relevant such a procedure could be enough. Dyadic type families on a measure space $(X, \mu)$ are the basic examples of increasing sequences of $\sigma$-algebras on which orthonormal bases for $L^{2}(X, \mu)$ can be constructed (see [7]). Nevertheless, to our purpose, which makes use of some deep facts of harmonic analysis such as vector valued Fefferman-Stein inequality for the Hardy-Littlewood maximal operator, some inner and outer metric control of the sizes of the dyadic sets is important. A construction of this type on a space of homogeneous type, $X$, is given by M.Christ in [3]. The first step in Christ's construction is to introduce a tree structure on an index set $\mathcal{A}$ that is closely related to the metric structure on $X$. In other words, M.Christ defines a partial order on $\mathcal{A}$ satisfying some tree properties controlled by the distance. For details see Section 5 . The second step in [3] is the construction of a dyadic family, $\mathcal{D}$, based in the partial order defined on $\mathcal{A}$. In the definition of partial order the choice of ancestors in the tree is not unique at all. This diversity translates into a corresponding diversity of different families $\mathcal{D}$. We shall use $\mathfrak{D}$ for denote all these dyadic families. The next step in the construction of wavelet bases in the general context of space of homogeneous type is given by $\mathrm{H}$. Aimar in [1] (see also [2]). Starting with dyadic cubes of a Christ's family $\mathcal{D}$, in [1] a structure of generalized multiresolution analysis and a Haar system $\mathcal{H}$ controlled by the quasi distance $d$ are constructed. This construction provides also a variety of different Haar systems $\mathcal{H}$ associated to the same family $\mathcal{D}$. We shall use $\mathcal{H}(\mathcal{D})$ to denote all these Haar systems $\mathcal{H}$. Hence, it seems to us that a natural problem is to see whether or not all these systems $\mathcal{H}$ in $\bigcup_{\mathcal{D} \in \mathfrak{D}} \mathcal{H}(\mathcal{D})$ are equivalent on Lebesgue spaces, in the usual sense of equivalence of Schauder bases of Banach spaces given by [13] or [9]. This problem is considered in an even more general situation in Section 3. More precisely we shall deal with a quite general notion of equivalence of dyadic families which allow us to prove equivalence of Haar systems built on them. We would like to point out that the main tool in the proof of our result, given in Section 4, is the vector valued Fefferman-Stein maximal inequality on spaces of homogeneous type.

\section{DyAdiC SySTEMS AND ASSOCIATED HAAR SYSTEMS ON SPACES OF HOMOGENEOUS TYPE}

Let us recall the basic concepts of the general theory of space of homogeneous type. Assume that $X$ is a set, a nonnegative symmetric function $d$ on $X \times X$ is called a quasi-distance if there exists a constant $K$ such that

$$
d(x, y) \leq K[d(x, z)+d(z, y)],
$$

for every $x, y, z \in X$, and $d(x, y)=0$ if and only if $x=y$.

We shall say that $(X, d, \mu)$ is a space of homogeneous type if $d$ is a quasi-distance on $X, \mu$ is a positive Borel measure defined on a $\sigma$-algebra of subsets of $X$ which contains the balls, and there exists a constant $A$ such that

$$
0<\mu(B(x, 2 r)) \leq A \mu(B(x, r))<\infty
$$

holds for every $x \in X$ and every $r>0$. 
The sets $\{(x, y) \in X \times X: d(x, y)<1 / n\}$ define a basis of a metrizable uniform structure on $X$. The balls $B(x, r)=\{y: d(x, y)<r\}$ form a basis of neighborhoods of $x$ for the topology induced by the uniform structure. A basic caveat comes from the fact that the $d$-balls are generally not open sets. Moreover, it is not difficult to give examples of quasi-distances for which some balls are not even Borel measurable subsets of $X$. Nevertheless in [10], R. Macias and C. Segovia prove that if $d$ is a quasi-distance on $X$, then there exist a distance $\rho$ and a number $\alpha \geq 1$ such that $d$ is equivalent to $\rho^{\alpha}$. Hence except for some simple changes of scales we can keep working with $\rho$ instead of $\rho^{\alpha}$. So that we shall assume along this paper that $d$ is actually a distance on $X$, in other words that $K=1$ in (2.1).

We shall say that a subset $E$ of $X$ is $\epsilon$-disperse for $\epsilon>0$ if $d(x, y) \geq \epsilon$ for every $x, y$ in $E$ with $x \neq y$.

Given a set $E$ in $X$ we shall write $\operatorname{diam}(E)$ to denote its diameter. In other words, $\operatorname{diam}(E)=\sup \{d(x, y): x \in E, y \in E\}$. On the other hand given an open and bounded set $E$ in $X$ we define its eccentricity by $\varepsilon(E)=\frac{r_{i}(E)}{r_{o}(E)}$, where $r_{i}(E)=$ $\sup \{r: B(x, r) \subseteq E$ for some $x \in E\}$ and $r_{o}(E)=\inf \{r: E \subseteq B(x, r)$ for some $x \in$ $X\}$.

In order to be able to apply Lebesgue Differentiation Theorem we shall also assume that continuous functions are dense in $L^{1}(X, \mu)$. Under the assumption of completeness for $(X, d)$ and regularity of the measure $\mu$ this density can be achieved (see [11] and [12]).

Even when the basic example of dyadic cubes and Haar functions is provided by the Euclidean space with its standard dyadic cubes and Haar functions, the geometric structure of the underlying space may also be quite different than that of $\mathbb{R}^{n}$. To illustrate these differences let us start by some special but representative examples concerning the basic set on which we can build Haar type bases. The first example is the restriction of the standard Haar system to $[0,1)$. The second example corresponds to a purely atomic space defined by the counting measure on $\mathbb{Z}$. A mixed situation is given in the third example where we consider a quite heterogeneous space of homogeneous type. The fourth could help to understand a somehow subtle definition of the relevant subfamily $\tilde{\mathcal{D}}$, given in section 3 , of a dyadic system given $\mathcal{D}$. Let us point out that each one of the spaces contained in these examples is a metric space of homogeneous type with the given measure and the standard Euclidean distance inherited from $\mathbb{R}$.

Example 1. Set $X_{1}=[0,1)$. In order to obtain a Haar type basis for $L^{2}\left(X_{1}\right)$, with respect to Lebesgue measure, we only have to add to the subsystem $\mathcal{H}_{1}=$ $\left\{h_{k}^{j} \in \mathcal{H}: j \in \mathbb{N}, k \in K_{j}=\left\{0, \ldots, 2^{j}-1\right\}\right\}$ the constant function $\chi_{[0,1)}$. In terms of the multirresolution analysis structure $([8]), \mathcal{H}$ is associated to the decomposition $L^{2}(\mathbb{R})=\bigoplus_{j \in \mathbb{Z}} W_{j}, \mathcal{H}_{1}$ instead is related to $L^{2}([0,1))=V_{0}^{1} \oplus\left(\bigoplus_{j \in \mathbb{Z}} W_{j}^{1}\right)$, where $V_{0}^{1}$ is the one dimensional space generated by the constant function $\chi_{[0,1)}$ and $W_{j}^{1}$ is the $2^{j}$-dimensional space generated by $\left\{h_{k}^{j}: k \in K_{j}\right\}$.

Example 2. Set $X_{2}=\mathbb{Z}$ be the set of all integers. The space $L^{2}(\mathbb{Z})$ with the counting measure is nothing but $l^{2}(\mathbb{Z})$, the set of all square summable sequences indexed on $\mathbb{Z}$. Notice that $l^{2}(\mathbb{Z})$ is isomorphic in the Hilbert sense to the subspace of $L^{2}(\mathbb{R})$ of those function which are constant between two consecutive integers. Hence if $\mathbf{h}_{k}^{j}: \mathbb{Z} \longrightarrow \mathbb{R}, j<0, k \in \mathbb{Z}$, is given by $\mathbf{h}_{k}^{j}(m)=h_{k}^{j}(m)$ where $h_{k}^{j} \in \mathcal{H}$ is 
the standard Haar function, we see that $\mathcal{H}_{2}=\left\{\mathbf{h}_{k}^{j}: j<0, k \in \mathbb{Z}\right\}$ is an orthonormal basis for $l^{2}(\mathbb{Z})$. Now $l^{2}(\mathbb{Z})=\bigoplus_{-\infty}^{j=-1} W_{j}^{2}$. In other words $V_{0}^{2}=V_{1}^{2}=V_{2}^{2}=\ldots$, since we have no further resolution for the scales $2^{-j}, j>0$, because locally the space reduce to points.

Example 3. Let $X_{3}=\mathbb{Z} \cup[0,1 / 2)$ be equipped with the usual distance and the measure which counts on $\mathbb{Z} \backslash\{0\}$ and is twice the length on the interval $[0,1 / 2)$. Precisely, if $E$ is a Borel set in $X_{3}, \mu(E)=\#(E \cap(\mathbb{Z} \backslash\{0\}))+2 m(E \cap[0,1 / 2))$ where \# is the cardinal and $m$ is the one dimensional Lebesgue measure. Now some regions of the space have unlimited resolution, some other reduce to points. In fact, dyadic sets in $X_{3}$ can be obtained by intersection of the standard dyadic intervals in $\mathbb{R}$ with $X_{3}$. If $I_{k}^{j}=\left[\frac{k}{2^{j}}, \frac{k+1}{2^{j}}\right)$, the dyadic cubes in $X_{3}$ are the sets $Q_{k}^{j}=I_{k}^{j} \cap X_{3}, j \in \mathbb{Z}, k \in \mathbb{Z}$. Notice that if $\mathcal{D}^{j}$ denotes the family $\left\{Q_{k}^{j}: k \in \mathbb{Z}\right\}$ of all dyadic cubes of $\mathrm{j}$-th level, then $\mathcal{D}^{0}=\mathcal{D}^{1}$. On the other hand, $\mathcal{D}^{j} \neq \mathcal{D}^{j+1}$ for $j \neq 0$. Hence if $V_{j}$ is the subspace of those function in $L^{2}\left(X_{3}, \mu\right)$ which are constant on each $Q_{k}^{j}, k \in \mathbb{Z}$, we certainly have that ... $V_{-2}^{3} \subset V_{-1}^{3} \subset V_{0}^{3}=V_{1}^{3} \subset V_{2}^{3} \subset \ldots$; so that the corresponding sequence of wavelet spaces $W_{j}^{3}$ such that $W_{j}^{3} \bigoplus V_{j}^{3}=V_{j+1}^{3}$ satisfies that $W_{0}^{3}=\{0\}$. Nevertheless no other $W_{j}^{3}, j \neq 0$ is trivial. Still a Haar type basis for $L^{2}\left(X_{3}, \mu\right)$ can be obtained from $\mathcal{H}$. In fact, since $W_{0}^{3}=\{0\}$ there is no wavelet for the scale $2^{0}=1$. When $j \geq 1$ the only region of the space showing resolution at the scale $2^{-j}$ is the interval $[0,1 / 2)$. Hence we take all the standard Haar functions supported in $[0,1 / 2)$. In other words, $A=\left\{h_{k}^{j}: j \geq 1, k=0,1, \ldots, 2^{j-1}-1\right\}$. For $j \leq-1$ we may use for $k \neq 0$ the functions in $\mathcal{H}_{2}$. In other words $B=\left\{\mathbf{h}_{k}^{j} \in \mathcal{H}_{2}: j<0, k \neq 0\right\}$. We only have to take special care of $k=0$ when $j \leq-1$. If this is the case we consider the function defined by $g_{0}^{j}(x)=h_{0}^{j}(m)$ if $x=m \in \mathbb{Z} \backslash\{0\}$ and $g_{0}^{j}(x)=h_{0}^{j}(0)$ if $x \in[0,1 / 2)$. It is no difficult to show that $\mathcal{H}_{3}=A \cup B \cup\left\{g_{0}^{j}: j \leq-1\right\}$ is an orthonormal system for $L^{2}\left(X_{3}, \mu\right)$.

Example 4. Let $X_{4}=\bigcup_{l \in \mathbb{Z}}\left[l, l+2^{-10}\right)$ be equipped with the usual distance and the measure $d \mu=2^{10} d x$. Let us consider the restriction to $X_{4}$ of the usual dyadic intervals of $\mathbb{R}$. For any nonpositive integer $j$ this space at the scale $2^{j}$ looks quite similar to the one considered in Example 2. In particular no dyadic cube of the $j^{\text {th }}$ generation has any offspring when $j=0,1, \ldots, 9$. Hence, as a set, the "dyadic cube" $\left[0,2^{-10}\right)$ belong to each generation $\mathcal{D}^{j}$, for $j=0,1, \ldots, 9$, but only as a member of the $10^{t h}$ generation it has some "non-trivial" offspring. This behavior is also reflected in the fact that in this space $X_{4}$ there are not Haar type wavelets at the scales $2^{j}$ with $j=0,1, \ldots, 9$, but for all the other values of $j \in \mathbb{Z}$ there exists exactly one wavelet supported at each dyadic cube. We shall denote with $\mathcal{H}_{4}$ this Haar system.

The actual success of Christ's construction (see Section 5 for details) is the proof of existence of dyadic families with a precise metric control on the eccentricity of the dyadic objets, on spaces of homogeneous type. Nevertheless our results hold true for general families of dyadic sets controled by the distance. Let us start by giving a definition of dyadic families on the metric space of homogeneous type $(X, d, \mu)$. 
Definition 2.1. The class $\mathfrak{D}(\delta)$ of all dyadic families. We say that $\mathcal{D}=$ $\bigcup_{j \in \mathbb{Z}} \mathcal{D}^{j}$ is a dyadic family on $X$ with parameter $\delta \in(0,1)$, briefly that $\mathcal{D}$ belong $\mathfrak{D}(\delta)$, if each $\mathcal{D}^{j}$ is a family of open subsets $Q$ of $X$, such that

(d.1) For every $j \in \mathbb{Z}$ the cubes in $\mathcal{D}^{j}$ are pairwise disjoints.

(d.2) For every $j \in \mathbb{Z}$ the family $\mathcal{D}^{j}$ covers almost all $X$ in the sense that $\mu(X-$ $\left.\bigcup_{Q \in \mathcal{D}^{j}} Q\right)=0$.

(d.3) If $Q \in \mathcal{D}^{j}$ and $i<j$, then there exists a unique $\tilde{Q} \in \mathcal{D}^{i}$ such that $Q \subseteq \tilde{Q}$.

(d.4) If $Q \in \mathcal{D}^{j}$ and $\tilde{Q} \in \mathcal{D}^{i}$ with $i \leq j$, then either $Q \subseteq \tilde{Q}$ or $Q \cap \tilde{Q}=\emptyset$.

(d.5) There exists a positive integer $N$ depending only on $A$ such that for every $j \in \mathbb{Z}$ and all $Q \in \mathcal{D}^{j}$ the inequalities $1 \leq \#\left(\left\{Q^{\prime} \in \mathcal{D}^{j+1}: Q^{\prime} \subseteq Q\right\}\right) \leq N$ hold.

(d.6) There exist three constants $a_{1}, a_{2}$ and $a_{3}$ such that for each $Q \in \mathcal{D}^{j}$ we have that $a_{1} \delta^{j} \leq \operatorname{diam}(Q) \leq a_{2} \delta^{j}$ and the uniform lower bound for the eccentricity $\varepsilon(Q) \geq a_{3}$.

Examples 3 and 4 show that in some regions or at some scales the space may keep dividing in pieces that become smaller and smaller. But it could also happen that at some regions, refining scales does not involve an actual refinement of the space. We are interested in the identification of those scales and places of partition which shall give rise to the Haar functions. Properties $(d .1)$ to $(d .6)$ allow us to obtain two properties which shall be crucial in the proof of our main result.

(d.7) The families $\tilde{\mathcal{D}}^{j}=\left\{Q \in \mathcal{D}^{j}: \#\left(\left\{Q^{\prime} \in \mathcal{D}^{j+1}: Q^{\prime} \subseteq Q\right\}\right)>1\right\}, j \in \mathbb{Z}$ are pairwise disjoints.

In fact, given a cube $Q \in \tilde{\mathcal{D}}^{j}$, from (d.1) we have that $Q \notin \mathcal{D}^{j+1}$. Moreover, $Q \notin \mathcal{D}^{j+n}$ for any positive integer $n$ and hence $Q \notin \tilde{\mathcal{D}}^{j+n}$.

(d.8) Set $\tilde{\mathcal{D}}=\bigcup_{j \in \mathbb{Z}} \tilde{\mathcal{D}}^{j}$. The function $\mathcal{J}: \tilde{\mathcal{D}} \longrightarrow \mathbb{Z}$ given by $Q \mapsto \mathcal{J}(Q)$ if $Q \in \tilde{\mathcal{D}}^{\mathcal{J}(Q)}$ is well defined.

In the sequel we shall make use of the following notation. Let $\mathcal{L}$ be the function defined on $\tilde{\mathcal{D}}$ taking values in the family of subsets of $\mathcal{D}$, given by $\mathcal{L}(Q)=\left\{Q^{\prime} \in\right.$ $\left.\mathcal{D}^{j+1}: Q^{\prime} \subseteq Q\right\}$ for $Q \in \tilde{\mathcal{D}}^{j}$ and $j \in \mathbb{Z}$. We shall say that the cube $Q$ is the first-ancestor of $Q^{\prime}$ if $Q^{\prime} \in \mathcal{L}(Q)$. Notice that from $(d .5), 1 \leq \#(\mathcal{L}(Q)) \leq N$.

Since we are interested in proving the equivalence of all Haar bases regardless its construction, we define in general what we mean by a Haar system associated to a dyadic family.

Definition 2.2. Haar system associated to $\mathcal{D} \in \mathfrak{D}(\delta)$. Let $\mathcal{D}$ be a dyadic family on $(X, d, \mu)$ such that $\mathcal{D} \in \mathfrak{D}(\delta)$. A system $\mathcal{H}$ of functions $h$ on $X$ is a Haar system associated to $\mathcal{D}$ if it satisfies

(h.0) Each $h \in \mathcal{H}$ is a simple Borel measurable real function on $X$.

(h.1) For each $h \in \mathcal{H}$ there exists a unique $j \in \mathbb{Z}$ and a cube $Q=Q(h) \in \tilde{\mathcal{D}}^{j}$ such that $\{x \in X: h(x) \neq 0\} \subseteq Q$, and this property does not hold for any cube in $\mathcal{D}^{j+1}$.

(h.2) For every $Q \in \tilde{\mathcal{D}}$ there exist exactly $M_{Q}=\#(\mathcal{L}(Q))-1 \geq 1$ functions $h \in \mathcal{H}$ such that (h.1) holds. We shall write $\mathcal{H}_{Q}$ to denote the set of all these functions $h$.

(h.3) For each $h \in \mathcal{H}$ we have that $\int_{X} h d \mu=0$. 
(h.4) For each $Q \in \tilde{\mathcal{D}}$ let $V_{Q}$ denote the vector space of all functions on $Q$ which are constant on each $Q^{\prime} \in \mathcal{L}(Q)$. Then the system $\left\{\frac{\chi_{Q}}{(\mu(Q))^{1 / 2}}\right\} \cup \mathcal{H}_{Q}$ is an orthonormal basis for $V_{Q}$.

It is easy to show, following the proof in [1] (see also [2]), that, given $\mathcal{D}$ in $\mathfrak{D}(\delta)$, it is allways possible to construct Haar systems supported on the elements $Q$ of $\tilde{\mathcal{D}}$. This means that there exist systems $\mathcal{H}$ of functions $h$ on $X$ satisfying (h.0) to (h.4). This construction is by no way unique. In fact it depends on the orthonormalization procedure and even on the order given to the indicator functions of the offspring of a fixed cube.

In the sequel, we shall use the notation $\|f\|_{p}$ for the norm $\left(\int_{X}|f|^{p} d \mu\right)^{1 / p}$ in $L^{p}(X, \mu), 1<p<\infty$. The $L^{\infty}$-norm of the function $f,\|f\|_{\infty}$, is defined, as usual, as the $\mu$-essential least upper bound of $f$.

From Definition 2.2, we obtain the following two properties of the elements $h$ in any Haar system $\mathcal{H}$ which shall be useful.

(h.5) For each $h \in \mathcal{H}$ there exist two d-balls $B_{*}(h) \subseteq B^{*}(h)$ with comparable radii such that $\chi_{B_{*}(h)}(x) \leq \frac{|h(x)|}{\|h\|_{\infty}} \leq \chi_{B^{*}(h)}(x)$ for every $x \in X$.

(h.6) For each $h \in \mathcal{H}$ there exists $Q^{*} \in \mathcal{L}(Q(h))$ such that

$$
\frac{1}{\sqrt{N \mu\left(Q^{*}\right)}} \chi_{Q^{*}}(x) \leq|h(x)| \leq \sum_{Q^{\prime} \in \mathcal{L}(Q(h))} \frac{1}{\sqrt{\mu\left(Q^{\prime}\right)}} \chi_{Q^{\prime}}(x) .
$$

In fact, it easy to see that (h.5) immediately follows from (d.6) and (h.4). To prove (h.6) notice that from (h.2) and (h.4) we have on one hand that $h=$ $\sum_{Q^{\prime} \in \mathcal{L}(Q(h))} a_{Q^{\prime}} \chi_{Q^{\prime}}$ with $a_{Q^{\prime}} \in \mathbb{R}$ and on the other, that $\|h\|_{2}^{2}=1$. Hence for each $Q^{\prime} \in \mathcal{L}(Q(h))$ we have

$$
\left|a_{Q^{\prime}}\right|^{2} \mu\left(Q^{\prime}\right) \leq \sum_{Q^{\prime \prime} \in \mathcal{L}(Q(h))}\left|a_{Q^{\prime \prime}}\right|^{2} \mu\left(Q^{\prime \prime}\right)=1 .
$$

So that, from the first inequality, each $\left|a_{Q^{\prime}}\right|$ is bounded above by $\mu\left(Q^{\prime}\right)^{-1 / 2}$ which proves the upper bound in (h.6). Since $\sum_{Q^{\prime \prime} \in \mathcal{L}(Q(h))}\left|a_{Q^{\prime \prime}}\right|^{2} \mu\left(Q^{\prime \prime}\right)=1$, from (d.5) we get, for at least one $Q^{*} \in \mathcal{L}(Q(h))$, that $\left|a_{Q^{*}}\right|^{2} \mu\left(Q^{*}\right) \geq 1 / N$.

\section{Equivalence of dyadic systems and of HaAr bases. The main Result}

Our purpose is to give sufficient conditions on two dyadic systems in such a way that all Haar systems defined on each one of them are equivalent in a way that we shall specify later. In the sequel two dyadic families $\mathcal{D}_{1}=\bigcup_{j \in \mathbb{Z}} \mathcal{D}_{1}^{j}$ and $\mathcal{D}_{2}=\bigcup_{j \in \mathbb{Z}} \mathcal{D}_{2}^{j}$ in $\mathfrak{D}(\delta)$ on $X$ are given. We shall denote by $Q$ the elements of $\mathcal{D}_{1}$, by $R$ those in $\mathcal{D}_{2}$, and by $\mathcal{J}_{i}$ the function in (d.8) for $\mathcal{D}_{i}, i=1,2$. The situation described in Examples 3 and 4 in Section 2 shows that it could happen that no wavelet occurs for some levels of resolution. Therefore, the conditions over the families $\mathcal{D}_{1}$ and $\mathcal{D}_{2}$ are given on the subfamilies $\tilde{\mathcal{D}}_{1}$ and $\tilde{\mathcal{D}}_{2}$. This lead us to the following definition of equivalent dyadic families. 
Definition 3.1. Equivalent dyadic families. We say that the two dyadic families $\mathcal{D}_{1}$ and $\mathcal{D}_{2}$ in $\mathfrak{D}(\delta)$ are equivalent, briefly $\mathcal{D}_{1} \sim \mathcal{D}_{2}$, if there exist a constant $c>0$ and a relation $\mathfrak{R} \subseteq \tilde{\mathcal{D}}_{1} \times \tilde{\mathcal{D}}_{2}$ such that

(i) for each $Q \in \tilde{\mathcal{D}}_{1}$ there exists $R \in \tilde{\mathcal{D}}_{2}$ such that $(Q, R) \in \mathfrak{R}$;

(ii) for each $R \in \tilde{\mathcal{D}}_{2}$ there exists $Q \in \tilde{\mathcal{D}}_{1}$ such that $(Q, R) \in \mathfrak{R}$;

(iii) $\left|\mathcal{J}_{1}(Q)-\mathcal{J}_{2}(R)\right| \leq c$ for all $(Q, R) \in \mathfrak{R}$ and

(iv) $d(Q, R)=\inf \{d(x, y): x \in Q, y \in R\} \leq c \delta^{\mathcal{J}_{1}(Q)}$ for all $(Q, R) \in \mathfrak{R}$.

Properties (i) and (ii) mean that the domain and the image of the relation $\mathfrak{R}$ are $\tilde{\mathcal{D}}_{1}$ and $\tilde{\mathcal{D}}_{2}$ respectively. Notice that even when the inequality in (iv) looks non-symetric because its right hand side contain only $\mathcal{J}_{1}(Q)$, from (iii) we see that $\delta^{\mathcal{J}_{1}(Q)} \sim \delta^{\mathcal{J}_{2}(R)}$. Thus, (iv) holds with $\mathcal{J}_{2}(R)$ instead of $\mathcal{J}_{1}(Q)$, perhaps with a different constant $c$. Hence, since $\mathfrak{R}^{-1}$ satisfies (i) to (iv), we see that $\sim$ is an equivalence relation on $\mathfrak{D}(\delta)$. Let us point out the geometrical meaning of this notion of equivalence of dyadic families. Since the parameter $j$ is a measure of the scale, the relation $\mathfrak{R}$ is giving a connection between the two descriptions provided by $\mathcal{D}_{1}$ and $\mathcal{D}_{2}$ of similar regions in the space $X$ at similar scales. In Section 5 we shall prove that all dyadic families built with Christ's method, corresponding to a given sequence of $\delta^{j}$-nets for a given $\delta>0$, are equivalent in the above sense. The following proposition leads us to think that the relation $\mathfrak{R}$ looks somehow like an "invertible function".

Proposition 3.2. Let $\mathcal{D}_{1}$ and $\mathcal{D}_{2}$ be two dyadic families in $\mathfrak{D}(\delta)$. Let $\mathfrak{R} \subseteq \tilde{\mathcal{D}}_{1} \times \tilde{\mathcal{D}}_{2}$ be a relation such that (iii) and (iv) hold with some constant $c$. Then, there exists a constant $\tilde{N}$ such that $\#\left(\left\{R \in \tilde{\mathcal{D}}_{2}:(Q, R) \in \mathfrak{R}\right\}\right) \leq \tilde{N}$ and $\#\left(\left\{Q \in \tilde{\mathcal{D}}_{1}:(Q, R) \in\right.\right.$ $\mathfrak{R}\}) \leq \tilde{N}$, for all cube $Q \in \tilde{\mathcal{D}}_{1}$ and all cube $R \in \tilde{\mathcal{D}}_{2}$ respectively.

Proof. The symmetry in our hypotheses allows us to prove only one of the two estimates. Let us show the first one. Let $Q$ be a given cube in $\tilde{\mathcal{D}}_{1}$. We shall consider the elements of $\tilde{\mathcal{D}}_{2}$ which are neighbors of $Q$ in a specific sense. Set $\mathcal{V}(Q, j)=\left\{R \in \tilde{\mathcal{D}}_{2}:(Q, R) \in \mathfrak{R}\right.$ and $\left.\mathcal{J}_{2}(R)=j\right\}$, for any $j \in \mathbb{Z}$. Notice that $\left\{R \in \tilde{\mathcal{D}}_{2}:(Q, R) \in \mathfrak{R}\right\}=\bigcup_{j \in \mathbb{Z}} \mathcal{V}(Q, j)$ and that, from (iii), if $\left|j-\mathcal{J}_{1}(Q)\right|>c$, then $\mathcal{V}(Q, j)=\emptyset$. Hence we only have to show that for $\left|j-\mathcal{J}_{1}(Q)\right| \leq c$, \#(V $\left.(Q, j)\right)$ is bounded by an absolute constant. But for each $Q \in \tilde{\mathcal{D}}_{1}$ and $R \in \tilde{\mathcal{D}}_{2}$ there exist, from (d.6), points $x_{Q}$ and $x_{R}$ in $X$ such that $B\left(x_{Q}, c \delta^{\mathcal{J}_{1}(Q)}\right) \subseteq Q$ and $B\left(x_{R}, c \delta^{\mathcal{J}_{2}(R)}\right) \subseteq R$ for some constant $c$. Thus, it is easy to see that there is a constant $\beta<1$ such that the set $\left\{x_{R}: R \in \mathcal{V}(Q, j)\right\}$ is $\beta \delta^{\mathcal{J}_{1}(Q)}$-disperse and is contained in a ball $B\left(x_{Q}, \gamma \delta^{\mathcal{J}_{1}(Q)}\right)$ for some constant $\gamma$. It is well known, see [4], that such a set in a space of homogeneous type has to be bounded above by some purely geometric constant.

Let $\mathcal{H}_{1}=\{h\}$ and $\mathcal{H}_{2}=\{\psi\}$ be two Haar systems associated to $\mathcal{D}_{1}$ and $\mathcal{D}_{2}$ respectively, with $\mathcal{D}_{1} \sim \mathcal{D}_{2}$. Let $Q(h)$ and $R(\psi)$ be the dyadic cubes in (h.1) for $h \in \mathcal{H}_{1}$ and $\psi \in \mathcal{H}_{2}$ respectively. We shall say that $\Psi$ is a selection function in $\mathcal{H}_{2}$ associated to $\mathfrak{R}$ if $\Psi: \mathcal{H}_{1} \longrightarrow \mathcal{H}_{2}$ and for every $h \in \mathcal{H}_{1}$ we have that $(Q(h), R(\Psi(h))) \in \mathfrak{R}$. Notice that from the properties of $\mathfrak{R}$ all these selection functions are connecting wavelets in one system to wavelets in the other with similar supports in scale and location. We shall denote by $\mathcal{S}_{1,2}$ the set of all such selection functions. We shall say that $h \in \mathcal{H}_{1}$ and $\psi \in \mathcal{H}_{2}$ are $\mathcal{S}_{1,2}$-related if there exists 
$\Psi \in \mathcal{S}_{1,2}$ such that $\Psi(h)=\psi$. Symmetrically we say that a function $\mathfrak{h}: \mathcal{H}_{2} \longrightarrow \mathcal{H}_{1}$ is a selection function in $\mathcal{H}_{1}$ associated to $\mathfrak{R}^{-1}$ if $(Q(\mathfrak{h}(\psi)), R(\psi)) \in \mathfrak{R}$. With $\mathcal{S}_{2,1}$ we denote the set of all such selection functions.

The next theorem is the main result for the Lebesgue spaces $L^{p}(X, \mu), 1<p<$ $\infty$. It shall be an immediate consequence of Theorem 3.5.

Theorem 3.3. Let $\mathcal{D}_{1}$ and $\mathcal{D}_{2}$ be two dyadic families on $(X, d, \mu)$ such that $\mathcal{D}_{1} \sim$ $\mathcal{D}_{2}$. Let $\mathcal{H}_{1}$ and $\mathcal{H}_{2}$ be two Haar system associated to $\mathcal{D}_{1}$ and $\mathcal{D}_{2}$ respectively. Then, for each $\mathfrak{R} \subseteq \tilde{\mathcal{D}}_{1} \times \tilde{\mathcal{D}}_{2}$ as in Definition 3.1, there exists a positive constant $C$ such that the inequalities

$$
\left\|\sum_{h \in F} \lambda_{h} h\right\|_{p} \leq C\left\|\sum_{h \in F} \lambda_{h} \Psi(h)\right\|_{p}
$$

and

$$
\left\|\sum_{\psi \in G} \nu_{\psi} \psi\right\|_{p} \leq C\left\|\sum_{\psi \in G} \nu_{\psi} \mathfrak{h}(\psi)\right\|_{p}
$$

hold for every finite subsets $F$ of $\mathcal{H}_{1}$ and $G$ of $\mathcal{H}_{2}$, every choice of sequences $\left(\lambda_{h}\right.$ : $\left.h \in \mathcal{H}_{1}\right)$ and $\left(\nu_{\psi}: \psi \in \mathcal{H}_{2}\right)$ of real numbers and every couple of selection functions $\Psi \in \mathcal{S}_{1,2}$ and $\mathfrak{h} \in \mathcal{S}_{2,1}$ associated to $\mathfrak{R}$.

Notice that if there exists $\Psi \in \mathcal{S}_{1,2}$ such that $\Psi$ is one to one and onto, using (3.2) with $\mathfrak{h}=\Psi^{-1}$ we obtain the result contained in the next statement.

Corollary 3.4. Let $\mathcal{H}_{1}$ and $\mathcal{H}_{2}$ be as in Theorem 3.3. Assume that in $\mathcal{S}_{1,2}$ there exists a one to one and onto selection function $\Psi$, then there exist two positive constants $C_{1}$ and $C_{2}$ such that

$$
C_{1}\left\|\sum_{h \in F} \lambda_{h} h\right\|_{p} \leq\left\|\sum_{h \in F} \lambda_{h} \Psi(h)\right\|_{p} \leq C_{2}\left\|\sum_{h \in F} \lambda_{h} h\right\|_{p}
$$

hold for every finite subset $F$ of $\mathcal{H}_{1}$ and every sequence $\left(\lambda_{h}: h \in \mathcal{H}_{1}\right)$ of real numbers.

The situation described in the above corollary applies to the case for different systems of Haar wavelets built on the standard dyadic cubes in Euclidean spaces. Moreover as we shall see in Section 5, this holds for two Haar systems built on two different, but equivalent, Christ's dyadic systems with the same $\delta^{j}$-nets.

Let us describe the result contained in Corollary 3.4 in terms of the standard notion of equivalence of vectors in a Banach space as given in [13] and [9]. Let $\mathcal{B}_{1}$ be any sequence containing once each element of $\mathcal{H}_{1}$, in other words, $\mathcal{B}_{1}=\left(h_{k}\right.$ : $\left.k \in \mathbb{Z}^{+}\right)$with $h_{k} \neq h_{j}$ if $k \neq j$ and $\mathcal{H}_{1}=\left\{h_{k}: k \in \mathbb{Z}^{+}\right\}$. Once the sequence $\mathcal{B}_{1}$ is chosen, with $\mathcal{B}_{2}=\left(\psi_{k}=\Psi\left(h_{k}\right): k \in \mathbb{Z}^{+}\right)$, we have that $\mathcal{H}_{2}=\left\{\psi_{k}: k \in \mathbb{Z}^{+}\right\}$, that $\psi_{k} \neq \psi_{j}$ if $k \neq j$ and that $\mathcal{B}_{1}$ and $\mathcal{B}_{2}$ are equivalent in the sense of [9]. In other words

$$
C_{1}\left\|\sum_{k \in F} \lambda_{k} h_{k}\right\|_{p} \leq\left\|\sum_{k \in F} \lambda_{k} \psi_{k}\right\|_{p} \leq C_{2}\left\|\sum_{k \in F} \lambda_{k} h_{k}\right\|_{p}
$$

for every finite subset $F$ of $\mathbb{Z}^{+}$and any scalar sequence $\left(\lambda_{k}: k \in \mathbb{Z}^{+}\right)$. 
The role played by properties (iii) and (iv) is crucial. In fact, it is known (see [9]) that a particular permutation of the Haar sequence is not equivalent to the Haar sequence itself.

Actually, since our basic tool, the vector valued Fefferman-Stein inequality for the Hardy-Littlewood maximal operator holds on weighted Lebesgue spaces and since the characterization of weighted Lebesgue spaces with Muckenhoupt weights also holds, we can obtain a weighted extension of Theorem 3.3.

Let us recall that a non-negative, measurable and locally integrable function $w$ defined on the space of homogeneous type $(X, d, \mu)$, is said to be a Muckenhoupt weight of class $A_{p}, 1<p<\infty$ if the inequality

$$
\left(\frac{1}{\mu(B)} \int_{B} w(x) d \mu(x)\right)\left(\frac{1}{\mu(B)} \int_{B} w(x)^{\frac{-1}{p-1}} d \mu(x)\right)^{p-1} \leq C
$$

holds for some constant $C$ and every ball $B$. With $\|f\|_{p, w}$ we shall denote the weighted Lebesgue norm of the function $f$, that is, $\|f\|_{p, w}=\left(\int|f|^{p} w d \mu\right)^{1 / p}$.

Theorem 3.5. Let $\mathcal{D}_{1}$ and $\mathcal{D}_{2}$ be two dyadic families on $(X, d, \mu)$ such that $\mathcal{D}_{1} \sim$ $\mathcal{D}_{2}$. Let $\mathcal{H}_{1}$ and $\mathcal{H}_{2}$ two Haar system associated to $\mathcal{D}_{1}$ and $\mathcal{D}_{2}$ respectively. Let $w$ be a Muckenhoupt weight of class $A_{p}, 1<p<\infty$. Then, for each $\mathfrak{R} \subseteq \tilde{\mathcal{D}}_{1} \times \tilde{\mathcal{D}}_{2}$ as in Definition 3.1, there exists a positive constant $C$ such that the inequalities

$$
\left\|\sum_{h \in F} \lambda_{h} h\right\|_{p, w} \leq C\left\|\sum_{h \in F} \lambda_{h} \Psi(h)\right\|_{p, w}
$$

and

$$
\left\|\sum_{\psi \in G} \nu_{\psi} \psi\right\|_{p, w} \leq C\left\|\sum_{\psi \in G} \nu_{\psi} \mathfrak{h}(\psi)\right\|_{p, w}
$$

hold for every finite subsets $F$ of $\mathcal{H}_{1}$ and $G$ of $\mathcal{H}_{2}$, every choice of sequences $\left(\lambda_{h}\right.$ : $\left.h \in \mathcal{H}_{1}\right)$ and $\left(\nu_{\psi}: \psi \in \mathcal{H}_{2}\right)$ of real numbers and every couple of selection functions $\Psi \in \mathcal{S}_{1,2}$ and $\mathfrak{h} \in \mathcal{S}_{2,1}$ associated to $\mathfrak{R}$.

Notice finally that Theorem 3.3 follows from Theorem 3.5 with $w \equiv 1$.

\section{Proof of Theorem 3.5}

The following theorem, which is a weighted extension of Fefferman-Stein inequality ([5]) to space of homogeneous type (see [6]), is one of the basic tools for the proof of Theorem 3.5. This result is a vector valued inequality for the Hardy-Litllewood maximal operator on $(X, d, \mu)$,

$$
M f(x)=\sup _{x \in B} \frac{1}{\mu(B)} \int_{B}|f(y)| d \mu(y),
$$

where the supremum is taken over the family of all $d$-balls in $X$ containing $x$. The result is contained in Theorem 6.6.2 in [6].

Theorem 4.1. Let $(X, d, \mu)$ be a metric space of homogeneous type such that continuous functions are dense in $L^{1}(X, \mu)$ and let $w$ be a Muckenhoupt weight of class $A_{p}, 1<p<\infty$. Then for each $1<r<\infty$ and for each $1<p<\infty$ there exists 
a constant $C_{r, p}$ depending only on $r, p, A$ and the $A_{p}$ constant of $w$ such that the inequality

$$
\left\|\left(\sum_{k=1}^{\infty}\left|M\left(f_{k}\right)\right|^{r}\right)^{1 / r}\right\|_{p, w} \leq C_{r, p}\left\|\left(\sum_{k=1}^{\infty}\left|f_{k}\right|^{r}\right)^{1 / r}\right\|_{p, w}
$$

holds for every sequence $\left\{f_{k}: k \in \mathbb{N}\right\}$ of real valued Borel measurable functions defined on $X$.

The second basic tool, which can be proved as in [2], is the following characterization of weighted Lebesgue spaces via Haar coefficients. We point out that we are stating the result in the precise form in which we shall use it in the proof of Theorem 3.5. In this way we avoid the somehow subtle distinction of cases according to the finiteness or not of the measure of the whole space $X$. In fact we shall use the next result only when $f$ is a linear combination of Haar wavelets.

Theorem 4.2. Let $\mathcal{H}$ be any Haar system associated to $\mathcal{D} \in \mathfrak{D}(\delta)$. Let $w$ be a Muckenhoupt weight of class $A_{p}, 1<p<\infty$. Then the system $\mathcal{H}$ is an unconditional basis of each $L^{p}(X, \mu), 1<p<\infty$. Moreover there exist two positive constants $C_{1}$ and $C_{2}$ such that for all $f \in L^{p}(X, \mu)$ with bounded support and vanishing integral

$$
C_{1}\|f\|_{p, w} \leq\left\|\left(\sum_{h \in \mathcal{H}}|<f, h>|^{2}|h|^{2}\right)^{1 / 2}\right\|_{p, w} \leq C_{2}\|f\|_{p, w} .
$$

We shall also use the following two technical lemmas. The first one provides a comparison of the maximum values of two related Haar functions corresponding to two equivalent dyadic systems. The second contains an elementary geometric consequence of the doubling property written in terms of the Hardy-Litllewood maximal operator.

Lemma 4.3. Let $\mathcal{D}_{1}$ and $\mathcal{D}_{2}$ be two dyadic families on $(X, d, \mu)$ such that $\mathcal{D}_{1} \sim \mathcal{D}_{2}$. Let $\mathcal{H}_{1}$ and $\mathcal{H}_{2}$ two Haar system associated to $\mathcal{D}_{1}$ and $\mathcal{D}_{2}$ respectively. Then, for each $\mathfrak{R} \subseteq \tilde{\mathcal{D}}_{1} \times \tilde{\mathcal{D}}_{2}$ as in Definition 3.1 there exists two positive constants $c$ and $C$ such that

$$
c \leq \frac{\|h\|_{\infty}}{\|\psi\|_{\infty}} \leq C,
$$

for every $h \in \mathcal{H}_{1}$ and every $\psi \in \mathcal{H}_{2}$ with $(Q(h), R(\psi)) \in \mathfrak{R}$.

Lemma 4.4. For every choice of positive constants $\alpha_{1}, \alpha_{2}, \alpha_{3}$ with $\alpha_{1} \leq \alpha_{2}$ there exists another constant $\beta \geq 1$, depending only on $\alpha_{i}, i=1,2,3$ and $A$ such that the inequality

$$
\chi_{B(y, r)}(x) \leq \beta M\left(\chi_{B(z, R)}\right)(x)
$$

holds for every $x \in X$, every $r$ and $R$ with $\alpha_{1} \leq \frac{R}{r} \leq \alpha_{2}$ and every $y, z \in X$ with $d(y, z)<\alpha_{3} r$.

We shall prove the above lemmas at the end of this section.

Proof of Theorem 3.5 Let us observe that it is enough to prove any one of the desired inequalities. We shall prove (3.3). First notice that since both dyadic families $\mathcal{D}_{1}$ and $\mathcal{D}_{2}$ belong to $\mathfrak{D}(\delta)$ and (iii) and (iv) hold, property (h.5) for the 
two Haar functions $h$ and $\Psi(h)$ corresponding to $\mathcal{H}_{1}$ and $\mathcal{H}_{2}$, respectively, with $\Psi \in \mathcal{S}_{1,2}$, involves balls which are all in the conditions of Lemma 4.4. In other words the four balls $B_{*}(h), B^{*}(h), B_{*}(\Psi(h)), B^{*}(\Psi(h))$, have comparable radii and the distance from one center to another is bounded by a constant times any one of those radii.

Let $F \subset \mathcal{H}$ finite, $\Psi \in \mathcal{S}_{1,2}$ and $\left(\lambda_{h}\right)$ a sequence of scalars. To avoid interruption in the next chain of seven inequalities involved in the proof of (3.3), we proceed to describe the arguments used in each one of them. In the first we apply the lower bound in Theorem 4.2 with $f=\sum_{h \in F} \lambda_{h} h$. For the second we use the upper bound in (h.5). The third comes from the upper bound for (4.1) in Lemma 4.3. The fourth is based in Lemma 4.4 and the positive homogeneity of the Hardy-Littlewood maximal operator. The fifth one is nothing but Fefferman-Stein inequality with $r=2$. The sixth is the lower bound in (h.5). The last one is the lower bound in Theorem 4.2 for $f=\sum_{h \in F} \lambda_{h} \Psi(h)$.

$$
\begin{aligned}
& \left\|\sum_{h \in F} \lambda_{h} h\right\|_{p, w} \leq C\left\|\left(\sum_{h \in F} \lambda_{h}^{2}|h|^{2}\right)^{1 / 2}\right\|_{p, w} \\
& \leq C\left\|\left(\sum_{h \in F} \lambda_{h}^{2}\|h\|_{\infty}^{2} \chi_{B^{*}(h)}\right)^{1 / 2}\right\|_{p, w} \\
& \leq C\left\|\left(\sum_{h \in F} \lambda_{h}^{2}\|\Psi(h)\|_{\infty}^{2} \chi_{B^{*}(h)}\right)^{1 / 2}\right\|_{p, w} \\
& \leq C\left\|\left(\sum_{h \in F}\left[M\left(\lambda_{h}\|\Psi(h)\|_{\infty} \chi_{B_{*}(\Psi(h))}\right)\right]^{2}\right)^{1 / 2}\right\|_{p, w} \\
& \leq C\left\|\left(\sum_{h \in F} \lambda_{h}^{2}\|\Psi(h)\|_{\infty}^{2} \chi_{B_{*}(\Psi(h))}\right)^{1 / 2}\right\|_{p, w} \\
& \leq C\left\|\left(\sum_{h \in F} \lambda_{h}^{2}|\Psi(h)|^{2}\right)^{1 / 2}\right\|_{p, w} \\
& \leq C\left\|\sum_{h \in F} \lambda_{k} \Psi(h)\right\|_{p, w} .
\end{aligned}
$$

The constants in the above inequalities may differ from one line to another.

Now we prove Lemmas 4.3 and 4.4 .

Proof of Lemma 4.3. From (h.6) applied to the system $\mathcal{H}_{1}$ we observe that the $L^{\infty}$-norm of the Haar function $h$ is equivalent to $\mu(Q(h))^{-1 / 2}$. For $\mathcal{H}_{2}$ instead, $\|\psi\|_{\infty}$ is of the order of $\mu(R(\psi))^{-1 / 2}$. Since all the constants in these equivalences depend only on the geometric constants and the two systems $\mathcal{D}_{1}$ and $\mathcal{D}_{2}$ are related 
by $\mathfrak{R}$, we get the desired equivalence of $\|h\|_{\infty}$ and $\|\psi\|_{\infty}$ when $(Q(h), R(\psi)) \in \mathfrak{R}$, with constants independent of $h$ and $\psi$.

Proof of Lemma 4.4. Notice first that there is nothing to prove when $x \notin$ $B(y, r)$ since the left hand side in the desired inequality vanishes. Assuming that $x \in B(y, r)$ we have $B(z, R) \subseteq B(x, \gamma r)$ with $\gamma=\alpha_{2}+\alpha_{3}+1$. In fact, if $u \in B(z, R)$ then

$$
\begin{aligned}
d(u, x) & \leq d(u, z)+d(z, y)+d(y, x) \\
& \leq R+\alpha_{3} r+r \leq \gamma r .
\end{aligned}
$$

Therefore

$$
M\left(\chi_{B(z, R)}\right)(x) \geq \frac{\mu(B(x, \gamma r) \cap B(z, R))}{\mu(B(x, \gamma r))}=\frac{\mu(B(z, R))}{\mu(B(x, \gamma r))} .
$$

Since $B(x, \gamma r) \subseteq B\left(z, \frac{\gamma+1+\alpha_{3}}{\alpha_{1}} R\right)$, the doubling property for $\mu$ finishes the proof of the Lemma.

\section{Christ's DyadiC Systems}

The aim of this section is to show that different dyadic families of M. Christ with the same basic nets of points but different orders are equivalent. Thus, as a consequence, we shall obtain that Haar bases built on such dyadic families are equivalent.

First we shall sketch the construction (see [3]) of the dyadic sets in the context of metric spaces of homogeneous type. We shall say that, for $\varepsilon>0, \mathcal{N}$ is an $\varepsilon$-net in $X$ if $\mathcal{N}$ is a maximal $\varepsilon$-disperse subset of $X$. That is, $d\left(x, x^{\prime}\right) \geq \varepsilon$ for every $x, x^{\prime} \in \mathcal{N}$ with $x \neq x^{\prime}$ and if $E$ is any other subset of $X$ strictly containing $\mathcal{N}$ then there exists $y, y^{\prime} \in E$ with $y \neq y^{\prime}$ such that $d\left(y, y^{\prime}\right)<\varepsilon$. It is easy to show that since $(X, d)$ supports the doubling measure $\mu$, then any $\varepsilon$-disperse subset $\mathcal{N}$ of $X$ is countable. Moreover $\mathcal{N}$ is finite if and only if $X$ is bounded. In the sequel we shall use the notation $\mathcal{N}=\left\{x_{k}: k \in K(\varepsilon)\right\}$ to denote the elements of the $\varepsilon$-net $\mathcal{N}$, where $K(\varepsilon)$ is an initial interval of positive integers, which could be the whole set $\mathbb{Z}^{+}$of positive integers. From the above remark $K(\varepsilon)=\mathbb{Z}^{+}$if and only if $X$ is unbounded. For a fixed positive $\delta$, the above construction with $\varepsilon=\delta^{j}, j \in \mathbb{Z}$, gives rise to a sequence of $\delta^{j}$-nets $\mathcal{N}_{j}=\left\{x_{k}^{j}: k \in K_{j}\right\}$, where $K_{j}=K\left(\delta^{j}\right)$. The set $\mathcal{A}=\left\{(j, k): j \in \mathbb{Z}\right.$ and $\left.k \in K_{j}\right\}$ plays a central role in Christ's construction. From the properties of dispersion and maximality of each net $\mathcal{N}_{j}$ the two following statements are easy to check for each $(j, k) \in \mathcal{A}$.

$(\alpha)$ There exists at most one $l_{0} \in K_{j-1}$ such that $d\left(x_{k}^{j}, x_{l_{0}}^{j-1}\right)<\frac{\delta^{j-1}}{2}$; and

$(\beta)$ there exists at least one $l \in K_{j-1}$ such that $d\left(x_{k}^{j}, x_{l}^{j-1}\right)<\delta^{j-1}$.

These properties allow to define partial orders $\preceq$ on $\mathcal{A}$, induced by the metric $d$ and the given sequence of $\delta^{j}$-nets in the following way:

I) $\quad$ take $(j, k) \in \mathcal{A}$

(a) if there exist $l_{0} \in K_{j-1}$ such that $d\left(x_{k}^{j}, x_{l_{0}}^{j-1}\right)<\frac{\delta^{j-1}}{2}$ then we decree that $(j, k) \preceq\left(j-1, l_{0}\right)$, 
(b) if there is not such an $l_{0} \in K_{j-1}$, we select any $l \in K_{j-1}$ for which $d\left(x_{k}^{j}, x_{l}^{j-1}\right)<\delta^{j-1}$, and decree that $(j, k) \preceq(j-1, l)$,

$I I)$ decree that $(j, k)$ is not related to any other $(j-1, s), s \in K_{j-1}$,

$I I I)$ extend $\preceq$ by transitivity.

For a given sequence of $\delta^{j}$-nets, $\mathcal{N}_{j}, \delta>0$, we shall say that such order belongs to the class $\mathcal{C}$, briefly, $\preceq \in \mathcal{C}$. We would like to emphasize at this point that since the $l$ provided by $(\beta)$ is far from being unique the selection in case (b) of the construction of $\preceq$ translates into a great diversity of possible orders satisfying all the desired properties. On the other hand, the case (a) in I) is giving the only rigidity of $\preceq$ that is reflected in the following property of the family $\mathcal{C}$.

$(\gamma)$ For each $(j, k) \in \mathcal{A}$ there exists al least one $u \in K_{j+1}$ such that $(j+1, u) \preceq$ $(j, k)$ for every order $\preceq \in \mathcal{C}$.

In fact, since $\delta<1 / 2$, from the maximality of $\mathcal{N}_{j+1}$, for each $(j, k) \in \mathcal{A}$ there exists $x_{u}^{j+1} \in \mathcal{N}_{j+1}$ such that $d\left(x_{u}^{j+1}, x_{k}^{j}\right)<\delta^{j+1}<\frac{\delta^{j}}{2}$. From the construction of $\preceq$ we obtain that $(j+1, u) \preceq(j, k)$ for every order $\preceq$.

Since the index $u \in K_{j+1}$ in $(\gamma)$ may not be unique, we shall select one of them, say $u=\mathfrak{b}(j, k)$. We shall say that $x_{\mathfrak{b}(j, k)}^{j+1}$ is the first-born from $x_{k}^{j}$.

For a given $\preceq \in \mathcal{C}$, the Christ dyadic cube at the level $j$ located at $k \in K_{j}$ is defined by

$$
Q_{k}^{j}=\bigcup_{(i, l) \preceq(j, k)} B\left(x_{l}^{i}, a \delta^{i}\right),
$$

which for some small values of the positive constant $a$ satisfy (d.1) to (d.8). The set $Q_{k}^{j}$ shall be called the dyadic cube associated to $x_{k}^{j} \in \mathcal{N}_{j}$. The family $\mathcal{D}_{\preceq}$ of all those $Q_{k}^{j}$ shall be called the Christ cubes associated to the family $\left\{\mathcal{N}_{j}\right.$ : $j \in \mathbb{Z}\}$ of nets and the order $\preceq$. Notice that different orders satisfying all the desired properties produce different shapes for the sets $Q_{k}^{j}$ corresponding to the level-position parameters $(j, k) \in \mathcal{A}$.

Given $\mathcal{D}_{i}=\mathcal{D}_{\preceq_{i}}$, where $\preceq_{i} \in \mathcal{C}$, we shall denote by $\tilde{\mathcal{D}}_{i}$ the family in $(d .7)$ for $\mathcal{D}_{i}$, by $Q$ the elements $Q_{k}^{j}$ of $\mathcal{D}_{1}$, by $R$ those cubes $R_{k}^{j}$ in $\mathcal{D}_{2}$, and by $\mathcal{J}_{i}$ the function in (d.8) for $\mathcal{D}_{i}$.

The main result in this section is contained in the next statement.

Theorem 5.1. Let $\preceq_{1}$ and $\preceq_{2}$ be two partial orders in $\mathcal{C}$ with $0<\delta<1 / 2$. Then $\mathcal{D}_{1} \sim \mathcal{D}_{2}$, where $\mathcal{D}_{i}=\mathcal{D}_{\preceq_{i}}, i=1,2$.

Its proof is an easy consequence of the following result.

Lemma 5.2. For every $Q \in \tilde{\mathcal{D}}_{1}$ there exist $R \in \tilde{\mathcal{D}}_{2}$ such that

$$
\mathcal{J}_{1}(Q)=\mathcal{J}_{2}(R) \text { and } d(Q, R) \leq 2 \delta^{\mathcal{J}_{1}(Q)} .
$$

Proof. Let $Q \in \tilde{\mathcal{D}}_{1}$ and $j=\mathcal{J}_{1}(Q)$. Thus, there exists $k \in K_{j}$ such that $Q=Q_{k}^{j}$. If $R_{k}^{j} \in \tilde{\mathcal{D}}_{2}$ then, since $x_{k}^{j} \in Q_{k}^{j} \cap R_{k}^{j}$, we obtain that (5.1) holds with $R=R_{k}^{j}$. Suppose now that $R_{k}^{j} \notin \tilde{\mathcal{D}}_{2}$. Set $\mathcal{O}_{1}=\left\{x_{l}^{j+1} \in \mathcal{N}_{j+1}:(j+1, l) \preceq_{1}\right.$ $(j, k)\}$ and $\mathcal{O}_{2}=\left\{x_{l}^{j+1} \in \mathcal{N}_{j+1}:(j+1, l) \preceq_{2}(j, k)\right\}$ to denote the offspring of $x_{k}^{j}$ in each order $\preceq_{i}$. From the construction of the cubes and since $R_{k}^{j} \notin \tilde{\mathcal{D}}_{2}$ 
we obtain that $\#\left(\mathcal{O}_{1}\right)>1$ and $\#\left(\mathcal{O}_{2}\right)=1$. Hence, $\mathcal{O}_{1} \backslash \mathcal{O}_{2} \neq \emptyset$. Otherwise $1=\#\left(\mathcal{O}_{2}\right) \geq \#\left(\mathcal{O}_{1}\right)>1$. Let us write $x_{s}^{j+1}$ to denote any element in $\mathcal{O}_{1} \backslash \mathcal{O}_{2}$. Thus, since $\mathcal{A}$ is a tree, there exist $(j, u) \in \mathcal{A}$ such that $(j+1, s) \preceq_{2}(j, u)$. Let $x_{\mathfrak{b}(j, u)}^{j+1}$ be the first-born associated to $(j, u) \in \mathcal{A}$. From $(\gamma),(j+1, s) \preceq_{1}(j, k)$ and we obtain that $x_{s}^{j+1} \neq x_{\mathfrak{b}(j, u)}^{j+1}$. Therefore $\#\left(\mathcal{O}_{2}\right)>1$. Hence, $R_{u}^{j} \in \tilde{\mathcal{D}}_{2}$. Moreover, $d\left(Q_{k}^{j}, R_{u}^{j}\right) \leq d\left(x_{k}^{j}, x_{s}^{j+1}\right)+d\left(x_{s}^{j+1}, x_{u}^{j}\right) \leq 2 \delta^{j}$ and (5.1) holds with $R=R_{u}^{j}$.

Proof of Theorem 5.1. The proof is an immediate consequence of the preceding lemma. In fact, let $\mathfrak{R}$ be the subset of $\tilde{\mathcal{D}}_{1} \times \tilde{\mathcal{D}}_{2}$ defined as the set of all couples $(Q, R)$ satisfying (5.1). Properties (iii) and (iv) in Definition 3.1 are contained in (5.1). Property (i) follows from the above lemma and property (ii) from the analogous to Lemma 5.2 changing the roles of $\tilde{\mathcal{D}}_{1}$ and $\tilde{\mathcal{D}}_{2}$.

In the sequel $\preceq_{1}$ and $\preceq_{2}$ are two partial orders in $\mathcal{C}$ as in Theorem 5.1. Let $\mathcal{H}_{1}=$ $\{h\}$ and $\mathcal{H}_{2}=\{\psi\}$ be two Haar systems associated with $\mathcal{D}_{1}$ and $\mathcal{D}_{2}$ respectively. Thus, from Theorem 3.5 we obtain that there exists a positive constant $C$ such that the inequalities

$$
\left\|\sum_{h \in F} \lambda_{h} h\right\|_{p, w} \leq C\left\|\sum_{h \in F} \lambda_{h} \Psi(h)\right\|_{p, w}
$$

and

$$
\left\|\sum_{\psi \in G} \nu_{\psi} \psi\right\|_{p, w} \leq C\left\|\sum_{\psi \in G} \nu_{\psi} \mathfrak{h}(\psi)\right\|_{p, w}
$$

hold for every finite subsets $F$ of $\mathcal{H}_{1}$ and $G$ of $\mathcal{H}_{2}$, every choice of sequences $\left(\lambda_{h}\right.$ : $\left.h \in \mathcal{H}_{1}\right)$ and $\left(\nu_{\psi}: \psi \in \mathcal{H}_{2}\right)$ of real numbers, every couple of selection functions $\Psi \in \mathcal{S}_{1,2}$ and $\mathfrak{h} \in \mathcal{S}_{2,1}$ associated to the relation $\mathfrak{R}$ given in the proof of Theorem 5.1 and every $w \in A_{p}$.

Moreover, we shall prove that there exists at least one function $\Psi: \mathcal{H}_{1} \longrightarrow \mathcal{H}_{2}$, one to one and onto that closely preserves scales and positions of the supports of the wavelets and therefore, we obtain that these Haar systems are equivalent bases on weighted Lebesgue spaces when these bases are related via $\Psi$. More precisely, we obtain the following result.

Proposition 5.3. There exists a one to one and onto function $\Psi: \mathcal{H}_{1} \longrightarrow \mathcal{H}_{2}$ such that for every $h \in \mathcal{H}_{1}$

$$
d(Q(h), R(\Psi(h)))=0 \quad \text { and } \mathcal{J}_{1}(Q(h))=\mathcal{J}_{2}(R(\Psi(h))),
$$

where, as before, $Q(h)$ is the cube in (h.1) for $h \in \mathcal{H}_{1}$ and $R(\psi)$ is the cube in (h.1) for $\psi \in \mathcal{H}_{2}$.

Proof. We shall first describe a special index set for the elements of $\mathcal{H}_{1}$ and $\mathcal{H}_{2}$. For each $(j, k) \in \mathcal{A}$ set $\mathcal{L}_{0}\left(Q_{k}^{j}\right)$ to denote the family of all cubes $Q_{l}^{j+1} \in \mathcal{D}_{1}^{j}$ such that $Q_{l}^{j+1} \subseteq Q_{k}^{j}$ and $Q_{l}^{j+1} \neq Q_{\mathfrak{b}(j, k)}^{j+1}$. Analogously, set $\mathcal{L}_{0}\left(R_{k}^{j}\right)$ to denote the family of all cubes $R_{l}^{j+1} \in \mathcal{D}_{2}^{j}$ such that $R_{l}^{j+1} \subseteq R_{k}^{j}$ and $R_{l}^{j+1} \neq R_{\mathfrak{b}(j, k)}^{j+1}$. We define the families $\mathcal{D}_{1}^{\prime}=\bigcup_{(j, k) \in \mathcal{A}} \mathcal{L}_{0}\left(Q_{k}^{j}\right)$ and $\mathcal{D}_{2}^{\prime}=\bigcup_{(j, k) \in \mathcal{A}} \mathcal{L}_{0}\left(R_{k}^{j}\right)$. From $(h .1)$ and $(h .2)$ for the system $\mathcal{H}_{1}$, we see that we can use $\mathcal{L}_{0}\left(Q_{k}^{j}\right)$ as an index set for the family of 
all the elements of $\mathcal{H}_{1}$ essentially supported on $Q_{k}^{j}$. Analogously, from (h.1) and (h.2) for the system $\mathcal{H}_{2}$, we can use $\mathcal{L}_{0}\left(R_{k}^{j}\right)$ as an index set for the family of all the elements of $\mathcal{H}_{2}$ essentially supported on $R_{k}^{j}$. Thus, $\mathcal{H}_{1}=\left\{h_{Q^{\prime}}: Q^{\prime} \in \mathcal{D}_{1}^{\prime}\right\}$ and $\mathcal{H}_{2}=\left\{\psi_{R^{\prime}}: R^{\prime} \in \mathcal{D}_{2}^{\prime}\right\}$. Notice that since from $(\gamma)$ the function $\mathfrak{b}(j, k)$ does not depend on the partial order in $\mathcal{C}$, we have that if $Q^{\prime}=Q_{m}^{l}$ is any element in $\mathcal{D}_{1}^{\prime}$, then $R_{m}^{l}=: R^{\prime}$ belongs to $\mathcal{D}_{2}^{\prime}$. Hence $\Psi: \mathcal{H}_{1} \longrightarrow \mathcal{H}_{2}$ given by $\Psi\left(h_{Q^{\prime}}\right)=\psi_{R^{\prime}}$ is well defined and one to one. On the other hand if $R^{\prime}=R_{m}^{l}$ is any element in $\mathcal{D}_{2}^{\prime}$, then $Q_{m}^{l}=: Q^{\prime}$ belongs to $\mathcal{D}_{1}^{\prime}$. So that $\Psi$ is onto $\mathcal{H}_{2}$. To finish the proof we check that $d\left(Q\left(h_{Q^{\prime}}\right), R\left(\Psi\left(h_{Q^{\prime}}\right)\right)\right)=0$ and $\mathcal{J}_{1}\left(Q\left(h_{Q^{\prime}}\right)\right)=\mathcal{J}_{2}\left(R\left(\Psi\left(h_{Q^{\prime}}\right)\right)\right)$. In fact, since $Q\left(h_{Q^{\prime}}\right)$ is the first ancestor of $Q^{\prime}=Q_{m}^{l}$ and $R\left(\Psi\left(h_{Q^{\prime}}\right)\right)=R\left(\psi_{R^{\prime}}\right)$ is the first ancestor of $R^{\prime}=R_{m}^{l}$ and $x_{m}^{l} \in Q_{m}^{l} \cap R_{m}^{l} \subseteq Q\left(h_{Q^{\prime}}\right) \cap R\left(\psi_{R^{\prime}}\right)$ we obtain that $d\left(Q\left(h_{Q^{\prime}}\right), R\left(\Psi\left(h_{Q^{\prime}}\right)\right)\right)=0$. On the other hand, both $Q\left(h_{Q^{\prime}}\right)$ and $R\left(\psi_{R^{\prime}}\right)$ belong to the same scale $\delta^{l-1}$, in other words $Q\left(h_{Q^{\prime}}\right) \in \mathcal{D}_{1}^{l-1}$ and $R\left(\psi_{R^{\prime}}\right) \in \mathcal{D}_{2}^{l-1}$. Hence $\mathcal{J}_{1}\left(Q\left(h_{Q^{\prime}}\right)\right)=\mathcal{J}_{2}\left(R\left(\Psi\left(h_{Q^{\prime}}\right)\right)\right)$.

Theorem 5.4. Let $\Psi: \mathcal{H}_{1} \longrightarrow \mathcal{H}_{2}$ be as in Proposition 5.3. Let $w$ be a Muckenhoupt weight of class $A_{p}, 1<p<\infty$. Then the bases $\mathcal{B}_{1}=\left(h: h \in \mathcal{H}_{1}\right)$ and $\mathcal{B}_{2}=\left(\psi_{h}=\Psi(h): h \in \mathcal{H}_{1}\right)$ are equivalent in weighted Lebesgue spaces. That is, there exist two positive constants $C_{1}$ and $C_{2}$ such that

$$
C_{1}\left\|\sum_{h \in F} \lambda_{h} h\right\|_{p, w} \leq\left\|\sum_{h \in F} \lambda_{h} \Psi(h)\right\|_{p, w} \leq C_{2}\left\|\sum_{h \in F} \lambda_{h} h\right\|_{p, w}
$$

hold for every finite subset $F$ of $\mathcal{H}_{1}$ and every sequence $\left(\lambda_{h}: h \in \mathcal{H}_{1}\right)$ of real numbers.

Proof. In fact, notice that since $\Psi$ satisfies that $(Q(h), R(\Psi(h))) \in \mathfrak{R}$ as defined in the proof of Theorem 5.1, if follows that $\Psi \in \mathcal{S}_{1,2}$. The result follows applying a weighted version of Corollary 3.4.

\section{REFERENCES}

[1] H. Aimar, Construction of Haar type bases on quasi-metric spaces with finite assouad dimension, Anal. Acad. Nac. Cs. Ex., F. y Nat., Buenos Aires 54 (2004).

[2] H. Aimar, A. Bernardis and B. Iaffei, Multiresolution approximation and unconditional bases on weighted Lebesgue spaces on spaces of homogeneous type, J. Approx. Theory, 148 (2007) 12-34.

[3] M. Christ, A T(b) theorem with remarks on analytic capacity and the Cauchy integral, Colloq. Math. 60/61 (2) (1990), 601-628.

[4] R. Coifman and G. Weiss, Analyse harmonique non-conmutative sur certains espaces homogenes, Lecture Notes in Math., 242, Springer-Verlag, Berlin 1971.

[5] C. Fefferman and E. M. Stein, Some maximal inequalities. Amer. J. Math. 93 (1971), $107-115$.

[6] I. Genebashvili, A. Gogatishvili, V. Kokilashvili and M. Krbec, Weight theory for integral transforms on space of homogeneous type, Addison Wesley Longman Limited. 1998.

[7] M. Girardi and W. Sweldens, A new class of unbalanced Haar wavelets that form an unconditional basis for $L_{p}$ on general measure spaces., J. Fourier Anal. Appl., 3(4) (1997) $457-474$.

[8] E. Hernandez and G. Weiss, A first course on wavelets, Studies in Advanced Mathematics, CRS PRESS, Boca Raton, 1996. 
[9] S. Konyagin and V. Temlyakov, A remark on greedy approximation in Banach spaces., East Journal on Approx., 5(3)(1999), 365-379.

[10] R. Macias and C. Segovia, Lipschitz functions on spaces of homogeneous type. Adv. in Math. 33 (1979), 271-309.

[11] K. Parthasarathy, Introduction to probability and measure, Springer-Verlag, New York 1978.

[12] R. Toledano, Desigualdades de Harnack elíptica y parabólica, un enfoque abstracto, Tesis doctoral. FIQ-UNL. 1999.

[13] R. Young, An introduction to nonharmonic Fourier series, Academic Press. 1980.

Departamento de Matemática (FIQ-UnL); IMAL-COnicet, Santa Fe, Argentina

E-mail address: haimar@santafe-conicet.gov.ar

Departamento de Matemática (FIQ-UnL); IMAl-COnicet, Santa Fe, Argentina

E-mail address: bernar@santafe-conicet.gov.ar

Departamento de Matemtica (FaEA-UnComa), Neuquen; Imal-COniCet, Santa Fe, Argentina

E-mail address: luisenlitoral@yahoo.com.ar 